\title{
Publisher Correction: The genomics of coloration provides insights into adaptive evolution
}

Anna Orteu (iD) and Chris D. Jiggins (iD)

Nature Reviews Genetics (2020) https://doi.org/10.1038/s41576-020-0234-z Published online 07 May 2020

In the article originally published online, the label for part c of Figure 3 was omitted. This error has been corrected in the HTML and PDF versions of the article.

https://doi.org/10.1038/s41576-020-0249-5 I Published online 18 May 2020

(c) Springer Nature Limited 2020 